\title{
Longitudinal Changes in the Oxygen Uptake Kinetic Response to Heavy-Intensity Exercise in 14- to 16-Year-Old Boys
}

\author{
Brynmor C. Breese, Craig A. Williams, Alan R. Barker, \\ and Joanne R. Welsman \\ University of Exeter
}

Samantha G. Fawkner

Heriot-Watt University

Neil Armstrong

University of Exeter

\begin{abstract}
This study examined longitudinal changes in the pulmonary oxygen uptake $\left(p \dot{V} \mathrm{O}_{2}\right)$ kinetic response to heavy-intensity exercise in 14-16 yr old boys. Fourteen healthy boys (age $14.1 \pm 0.2 \mathrm{yr}$ ) completed exercise testing on two occasions with a 2 -yr interval. Each participant completed a minimum of three 'step' exercise transitions, from unloaded pedalling to a constant work rate corresponding to $40 \%$ of the difference between the $p \dot{V} O_{2}$ at the gas exchange threshold and peak $p \dot{V} O_{2}(40 \% \Delta)$. Over the 2 -yr period a significant increase in the phase II time constant ( $25 \pm 5$ vs. $\left.30 \pm 5 \mathrm{~s} ; p=.002, \omega^{2}=0.34\right)$, the relative amplitude of the $p \dot{V} O_{2}$ slow component $\left(9 \pm 5\right.$ vs. $\left.13 \pm 4 \% ; p=.036, \omega^{2}=0.14\right)$ and the $p \dot{V} O_{2}$ gain at end-exercise $(11.6 \pm$ 0.6 vs. $\left.12.4 \pm 0.7 \mathrm{~mL} \cdot \mathrm{min}^{-1} \cdot \mathrm{W}^{-1} ; p<.001, \omega^{2}=0.42\right)$ were observed. These data indicate that the control of oxidative phosphorylation in response to heavy-intensity cycling exercise is age-dependent in teenage boys.
\end{abstract}

At the onset of a step transition in exercise there is an almost immediate increase in cardiac output which occurs before the arrival at the lungs of venous blood from the exercising muscles. This cardiodynamic phase (phase I) which lasts about 15-20s is independent of oxygen uptake at the muscle and reflects an increase in pulmonary blood flow with exercise. Phase II, the primary component, is a rapid exponential increase in pulmonary oxygen uptake $\left(p \dot{V} O_{2}\right)$ that arises with hypoxic

Breese, Williams, Barker, Welsman, and Armstrong are with the Children's Health and Exercise Research Center, University of Exeter, Exeter, EX1 2LU, UK. Fawkner is with the School of Life Sciences, Heriot-Watt University, Riccarton, Edinburgh, EH14 4AS, UK. 
and hypercapnic blood from the exercising muscles arriving at the lungs. Phase II kinetics are described by the time constant $(\tau)$ which is the time taken to achieve $63 \%$ of the change in $p \dot{V} O_{2}$. In phases I and II, adenosine triphosphate (ATP) resynthesis cannot be fully supported by oxidative phosphorylation and the additional energy requirements of the exercise are met from oxygen stores, phosphocreatine (PCr) and anaerobic glycolysis.

During moderate-intensity exercise [i.e., exercise below the gas exchange threshold (GET)], $p \dot{V} O_{2}$ reaches a steady state in children within about 2 min with an oxygen cost (gain) of about $10 \mathrm{~mL} \cdot \mathrm{min}^{-1} \cdot \mathrm{W}^{-1}$ above that found during unloaded pedalling. During heavy-intensity exercise [i.e., exercise above the GET but below critical power or maximal lactate steady state], the primary phase II gain is similar to that observed during moderate-intensity exercise but the oxygen cost increases over time as a slow component of $p \dot{V} O_{2}$ (phase III) is superimposed upon the primary component and the achievement of a steady state is delayed.

Recent studies using ${ }^{31} \mathrm{P}$-magnetic resonance spectroscopy $\left({ }^{31} \mathrm{P}-\mathrm{MRS}\right)$ have revealed a close kinetic coupling between muscle $\mathrm{PCr}$, a surrogate of muscle oxygen consumption $\left(m \dot{V} \mathrm{O}_{2}\right)$, and $p \dot{V} \mathrm{O}_{2}$ during both moderate-intensity and heavy-intensity exercise in adults $(30,31)$ and moderate-intensity exercise in children (4). The mechanisms underlying the slow component remain speculative but appear to be a function of muscle fiber distribution, motor unit recruitment and the matching of oxygen delivery to active muscle fibers (18). Therefore, the study of $p \dot{V} O_{2}$ kinetics to an imposed heavy-intensity exercise stimulus has the potential to provide important information on developmental changes in exercise metabolism.

Armon et al. (1) were the first to examine child-adult differences in $p \dot{V} O_{2}$ kinetics during 6 min of cycling exercise across a range of exercise intensities above the GET. They concluded that, unlike adults, a single exponential was sufficient to describe the $p \dot{V} O_{2}$ responses of a mixed group of boys and girls. Although their methodology of using an arbitrary linear term set from 3 to 6 min is likely to have misrepresented the actual magnitude of the $p \dot{V} O_{2}$ slow component $(13,14)$, Armon et al. did report a positive linear term in $73 \%$ of the children's responses above the GET. They observed children to exhibit faster exercise onset $p \dot{V} O_{2}$ kinetics and to consume a greater percentage of the final $p \dot{V} O_{2}$ during the initial exponential phase compared with adults (1). Williams et al. (36) also reported a negligible $p \dot{V} O_{2}$ slow component, comprising $0.9 \%$ of the total $p \dot{V} O_{2}$ response, in children during 6 min of heavy intensity treadmill running, and speculated that a single exponential model was adequate to derive the kinetic response parameters. However, given that the same authors reported that the $p \dot{V} O_{2}$ slow component is markedly reduced during treadmill running compared with cycling exercise (9), it is difficult to ascertain whether this finding is reflective of the child's physiology or the exercise modality employed.

To overcome these methodological concerns, Fawkner and Armstrong (13) longitudinally investigated the $p \dot{V} O_{2}$ kinetic response during heavy-intensity cycling exercise in prepubertal children over a 2 -yr period. These authors used an iterative curve fitting procedure to first identify the onset of the $p \dot{V} O_{2}$ slow component, and then fit a single exponential model to the phase II response only. In contrast to earlier reports, Fawkner and Armstrong reported a discernible $p \dot{V} \mathrm{O}_{2}$ slow component in prepubertal children, and, over the 2 -yr interval, found both the phase II $\tau$ and $p \dot{V} O_{2}$ slow component to significantly increase (13). Fawkner and Armstrong were the first to report the $95 \%$ confidence intervals of children's phase II $\tau$ during heavy-intensity exercise and to eliminate possible confounding effects of 
intersubject variability by using a longitudinal design to study appropriately agerelated influences on $p \dot{V} O_{2}$ kinetics in children. The results from this study support an age-dependent control of oxidative metabolism in 11-13 yr old children during heavy-intensity cycling exercise (13).

There are few data on $p \dot{V} O_{2}$ kinetics during heavy-intensity exercise in older children. A recent cross-sectional investigation reported that both the phase II $\tau$ and $p \dot{V} \mathrm{O}_{2}$ slow component during heavy-intensity cycling exercise in male adolescents aged 14-17 yrs were comparable to adult data reported by others (24). However, no previous investigation has examined whether the age-related changes in $p \dot{V} O_{2}$ kinetics during heavy-intensity exercise evidenced in prepubertal children (13) persist or increase through the teen years. Therefore, the purpose of this study was to longitudinally investigate changes in the $p \dot{V} O_{2}$ kinetic response to heavy-intensity cycling exercise in 14-16 yr old boys. We hypothesized that both the phase II $\tau$ and $p \dot{V} O_{2}$ slow component would significantly increase over a 2 -yr period.

\section{Methods}

\section{Participants}

Fourteen healthy boys completed all tests on two occasions, separated by a 2-yr interval. Written, informed consent was obtained from each participant and their parents. Ethical approval was granted by the Institutional Research Ethics Committee. Participants were instructed to visit the laboratory on a minimum of four occasions over a 2-wk period, and testing took place at approximately the same time of day for each participant. On each visit, participants' stature was measured with a Seca 220 stadiometer (Vogel \& Halke, Hamburg, Germany) and body mass determined by use of Seca electronic scales (Vogel \& Halke). Due to the prevailing sociological climate in the UK at the time of the study regarding maturity screening we were unable to assess the participants' maturational status.

\section{Experimental Procedures}

The data were collected using the same apparatus on both test occasions. All exercise tests were performed on an electronically- braked cycle ergometer (Lode Excalibur Sport, Groningen, the Netherlands) with the seat height, handlebar height, and crank length adapted to each child and subsequently maintained throughout the testing period. The Lode ergometer was calibrated according to the manufacturer's recommendations and had a baseline pedalling resistance equivalent to $10 \mathrm{~W}$ at 70 rpm. During all exercise tests, gas-exchange variables were measured and displayed online by use of an EX670 mass spectrometer and analysis suite (Morgan Medical, Rainham, UK) that was calibrated according to the manufacturer's instructions. Expired volume was measured using a turbine flowmeter (Interface Associates) with a dead space volume of $90 \mathrm{~mL}$. Volume calibration was achieved by using a handheld 3-L calibration syringe (Hans Rudolph, Kansas City, MO) over a range of flow speeds. The sum of the gas-transport and analyzer-response delay terms was determined, and appropriate adjustments were made in the software. All calibration procedures were repeated before each experimental test.

On the first visit, participants completed an incremental ramp test to voluntary exhaustion for determination of peak $p \dot{V} O_{2}$ and GET. After a 3-min warm-up 
of unloaded pedalling, the resistance increased continuously by either 25 or 40 $\mathrm{W} \cdot \mathrm{min}^{-1}$ to attain a test approximately $8-10 \mathrm{~min}$ in duration. Participants pedalled at a cadence of $70 \pm 5 \mathrm{rpm}$, and were actively encouraged to continue until voluntary exhaustion. Maximal effort was considered to have been given if, in addition to subjective indications of intense effort (e.g., excessive hyperpnoea, facial flushing, sweating, discomfort), respiratory exchange ratio reached a value $>1.00$. All participants satisfied these criteria. Peak $p \dot{V} O_{2}$ was taken as the highest recorded 10-s stationary average value during the maximal exercise test. The GET was determined noninvasively as the first disproportionate increase in $\mathrm{CO}_{2}$ production $\left(\dot{\mathrm{VCO}}_{2}\right)$ relative to the increase in $p \dot{V} \mathrm{O}_{2}$ using methods described in previous investigations from our laboratory (17).

On subsequent visits, subjects completed a series of 'step' exercise transitions consisting of $6 \mathrm{~min}$ of unloaded pedalling, followed instantaneously by a work rate that, by extrapolation from the ramp response, corresponded to $40 \%$ of the difference between the $p \dot{V} \mathrm{O}_{2}$ at the GET and peak $p \dot{V} \mathrm{O}_{2}(40 \% \Delta)$ for $9 \mathrm{~min}$. This intensity has been shown with boys to lie within the heavy exercise domain (12). A pedal cadence of $70 \mathrm{rpm}$ was maintained throughout. A single transition was completed on each visit and at least three and in most cases four transitions were completed in total to obtain $95 \%$ confidence intervals in the phase II $\tau$ of $\pm 5 \mathrm{~s}$.

\section{Data Analysis Procedures}

The breath-by-breath $p \dot{V} O_{2}$ data from each 'step' exercise were initially examined to exclude errant breaths by removing values lying more than four standard deviations from the local mean determined using a 5-breath rolling average. Filtered $p \dot{V} O_{2}$ data were subsequently linearly interpolated to provide second-by-second values and, for each individual, identical repetitions of each rest-to-exercise transition were time aligned to the start of exercise and averaged together to form a single data set for analysis.

The first $20 \mathrm{~s}$ of data after the onset of exercise were deleted to remove the phase I response, and a nonlinear least-square algorithm was used to fit the data described in the following single-exponential equation $(E q .1)$ :

$$
\Delta p \dot{V} O_{2}(t)=A \cdot\left(1-\mathrm{e}^{-(\mathrm{t}-\delta) / \tau}\right) \quad(\text { phase } 1<\mathrm{t}<540 \mathrm{~s})
$$

where $p \dot{V} O_{2}(t)$ is the increase in $p \dot{V} O_{2}$ at time $t$ above the prior control level (calculated as the mean $p \dot{V} O_{2}$ during the baseline phase of unloaded pedalling, omitting the first $180 \mathrm{~s}$ and the last $30 \mathrm{~s}$ ); $A, \tau$, and $\delta$ are the amplitude, time constant, and time delay respectively. To identify an optimal fitting window with which to estimate parameters of the phase II component, a purpose-designed software program developed with LabVIEW (National Instruments, Newbury, UK) was used to remove the possible influence of arbitrarily parametizing the slow component on the dependent parameters of the primary component. The fitting window was iteratively widened by $1 \mathrm{~s}$ intervals, starting from a $60 \mathrm{~s}$ fitting window and finishing with a fitting window that encompassed the entire data set (14). The estimated $\tau$ for each fitting window was plotted against time to allow the beginning of the slow component to be determined through visual inspection. The onset of the slow component was defined as the point at which a plateau in the estimated $\tau$ was followed by a progressive increase in the estimated $\tau$. 
The time constant $(\tau)$, delay term $(\delta)$, and amplitude $(A)$ and their $95 \%$ confidence intervals were subsequently estimated using the predetermined fitting window (GraphPad Prism, GraphPad Software, San Diego, CA). The amplitude of the slow component $\left(A_{s}\right.$ ') was calculated as the difference in the mean of the $p \dot{V} O_{2}$ amplitude over the last $30 \mathrm{~s}$ of exercise $\left(\Delta p \dot{V} O_{2} t o t\right)$ and $A$. The slow component was expressed in relative terms as the percentage contribution of $A_{s}$ ' to $\Delta p \dot{V} O_{2}$ tot. The functional 'gain' of the phase II $p \dot{V} O_{2}$ response was computed by dividing the amplitude of the phase II response $(A)$ by the change in work rate $(\Delta \mathrm{WR})$. This method was replicated to compute the functional gain of the entire response.

\section{Statistical Analyses}

Descriptive statistics (means $\pm S D$ ) were initially determined for variables of interest. Paired samples $t$ tests were used to identify significant changes in response measures between the two test occasions. Statistical significance was set at an alpha level of 0.05 . Omega squared $\left(\omega^{2}\right)$ statistics were used to investigate the effect size of any differences between test occasions.

\section{Results}

Participants' peak exercise responses to the ramp test are presented in Table 1. There was a significant increase in absolute values for peak $p \dot{V} O_{2}$ and GET over the 2-yr study period. No significant changes were found for the GET expressed as a percentage of peak $p \dot{\mathrm{V} O} \mathrm{O}_{2}$.

A representative response for a participant on each test occasion is illustrated in Figure 1, with the mean response profile for all participants on each test occasion shown in Figure 2. Table 2 presents the $p \dot{V} O_{2}$ kinetic parameters over time. Absolute $p \dot{V} O_{2}$ values for the phase II and end-exercise amplitudes significantly increased with age $\left(p=.002\right.$, effect size $\omega^{2}=0.35$, and $p=.001$, effect size $\omega^{2}=0.41$ respectively). There was a significantly longer phase II time constant $\left(p=.002\right.$, effect size $\omega^{2}=$ $0.34)$ and greater relative slow component amplitude $\left(p=.036\right.$, effect size $\left.\omega^{2}=0.14\right)$

Table 1 Physical Characteristics and Peak Exercise Responses on Two Separate Visits Over a 2-Year Interval

\begin{tabular}{lccc}
\hline & Test Occasion 1 & Test Occasion 2 & P value $\left(\omega^{2}\right)$ \\
\hline Age $(\mathrm{yr})$ & $14.1 \pm 0.2$ & $16.0 \pm 0.2$ & $0.001(1.00)$ \\
Body mass $(\mathrm{kg})$ & $62.9 \pm 10.2$ & $71.6 \pm 11.1$ & $0.001(0.48)$ \\
Stature $(\mathrm{m})$ & $1.72 \pm 0.10$ & $1.80 \pm 0.11$ & $0.001(0.72)$ \\
Peak $p \dot{V} O_{2}\left(\mathrm{~L} \cdot \mathrm{min}^{-1}\right)$ & $3.14 \pm 0.46$ & $3.78 \pm 0.41$ & $0.001(0.61)$ \\
GET $\left(\mathrm{L} \cdot \mathrm{min}^{-1}\right)$ & $1.56 \pm 0.25$ & $1.91 \pm 0.33$ & $0.003(0.39)$ \\
$\mathrm{GET}\left(\%\right.$ peak $\left.p \dot{V} \mathrm{O}_{2}\right)$ & $50 \pm 5$ & $49 \pm 3$ & $0.712(0.04)$ \\
$p \dot{V} \mathrm{O}_{2}$ at $40 \% \Delta\left(\mathrm{L} \cdot \mathrm{min}^{-1}\right)$ & $2.20 \pm 0.32$ & $2.66 \pm 0.34$ & $0.001(0.57)$ \\
$p \dot{V} \mathrm{O}_{2}$ at $40 \% \Delta\left(\%\right.$ peak $\left.p \dot{V} \mathrm{O}_{2}\right)$ & $70 \pm 3$ & $70 \pm 3$ & $0.786(0.04)$ \\
\hline
\end{tabular}

Note. Values are mean $\pm \mathrm{SD}$. 


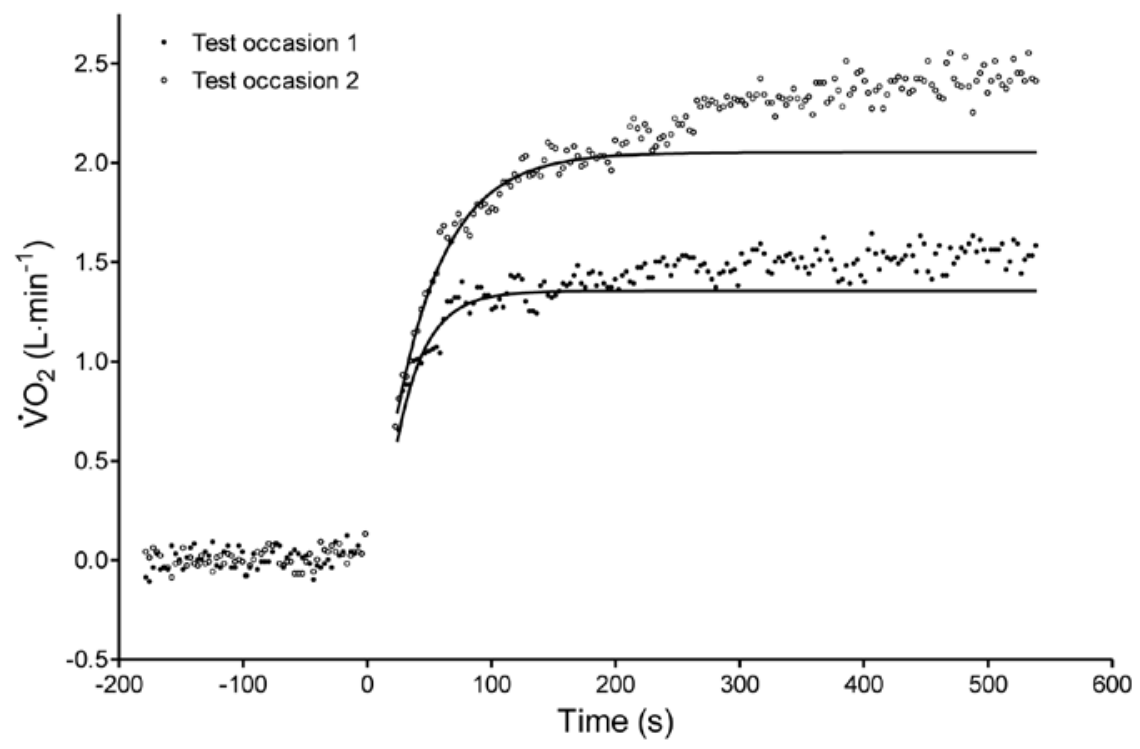

Figure 1 - Schematic of the $p \dot{V} O_{2}$ response in a representative participant to a step change in exercise work rate, on each test occasion. The single exponential fit defining the predicted $\mathrm{O}_{2}$ cost of exercise is extended to demonstrate the magnitude of the $p \dot{V} O_{2}$ slow component. The phase 1 portion of the $p \dot{V} O_{2}$ response is removed for clarity.

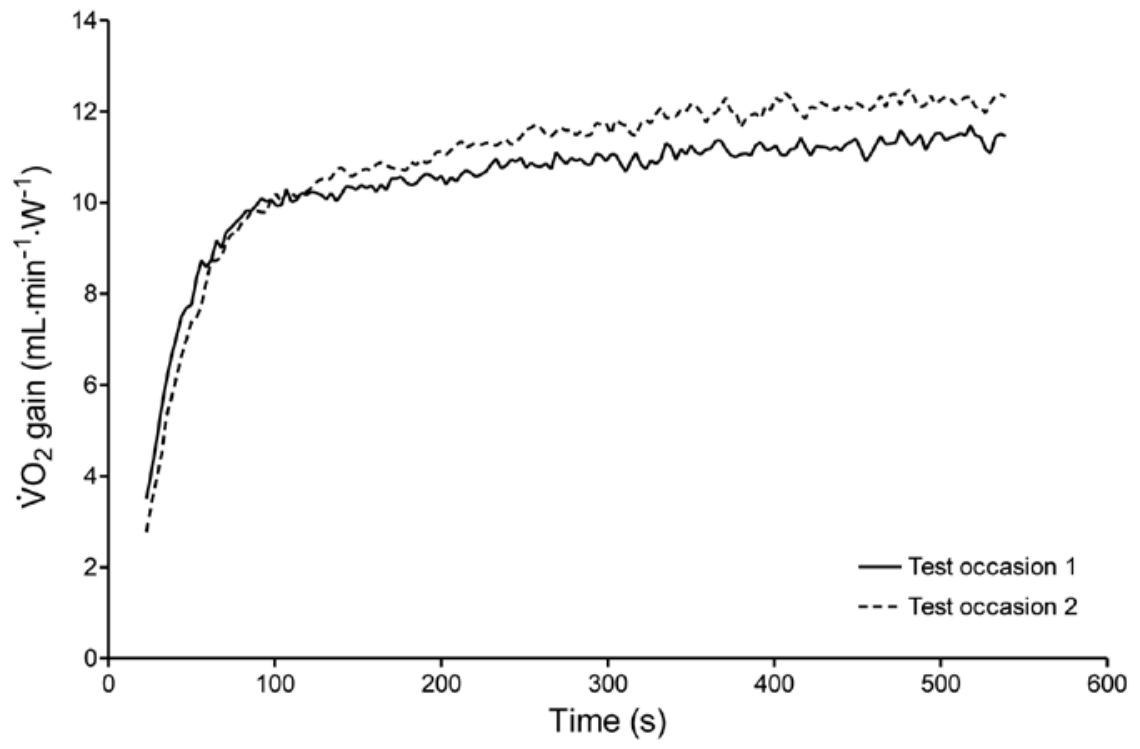

Figure 2 - Mean temporal profile of the gain of the $p \dot{V} \mathrm{O}_{2}$ response to heavy-intensity exercise on each test occasion. The phase 1 portion of the $p \dot{V} O_{2}$ response is removed for clarity. 
Table $2 \quad p \mathrm{VVO}_{2}$ Kinetic Response Parameters on Two Separate Visits Over a 2-Year Interval

\begin{tabular}{lccc}
\hline & Test Occasion 1 & Test Occasion 2 & P value $\left(\omega^{2}\right)$ \\
\hline Phase II time delay $(\mathrm{s})$ & $12 \pm 3$ & $13 \pm 4$ & $0.232(0.02)$ \\
Phase II $\tau(\mathrm{s})$ & $25 \pm 5$ & $30 \pm 5$ & $0.002(0.34)$ \\
Phase II amplitude $\left(\mathrm{L} \cdot \mathrm{min}^{-1}\right)$ & $1.56 \pm 0.34$ & $1.92 \pm 0.28$ & $0.002(0.35)$ \\
Phase II gain $\left(\mathrm{mL} \cdot \mathrm{min}^{-1} \cdot \mathrm{W}^{-1}\right)$ & $10.5 \pm 0.8$ & $10.8 \pm 0.7$ & $0.157(0.04)$ \\
$p \dot{V} O_{2}$ slow component $\left(\mathrm{L} \cdot \mathrm{min}^{-1}\right)$ & $0.16 \pm 0.08$ & $0.28 \pm 0.10$ & $0.003(0.30)$ \\
Relative $p \dot{V} O_{2}$ slow component $(\%)$ & $9 \pm 5$ & $13 \pm 4$ & $0.036(0.14)$ \\
End-exercise $p \dot{V} O_{2}\left(\mathrm{~L} \cdot \mathrm{min}^{-1}\right)$ & $1.72 \pm 0.38$ & $2.20 \pm 0.32$ & $0.001(0.41)$ \\
End-exercise gain $\left(\mathrm{mL} \cdot \mathrm{min}^{-1} \cdot \mathrm{W}^{-1}\right)$ & $11.6 \pm 0.6$ & $12.4 \pm 0.7$ & $0.001(0.42)$ \\
\hline
\end{tabular}

Note. Values are mean $\pm \mathrm{SD}$

on test occasion 2. No significant change in the gain of $p \dot{V} O_{2}$ relative to work rate $\left(\mathrm{mL} \cdot \mathrm{min}^{-1} \cdot \mathrm{W}^{-1}\right)$ was found over the phase II period. However, the end-exercise $p \dot{V} O_{2}$ gain was significantly greater on test occasion $2\left(p=.000\right.$, effect size $\left.\omega^{2}=0.42\right)$.

\section{Discussion}

This is the first study to investigate longitudinally the $p \dot{V} O_{2}$ kinetic response at the onset of heavy-intensity cycling exercise in teenage boys. The principal findings are that over a 2-yr interval: i) the phase II $\tau$ significantly slowed by approximately $5 \mathrm{~s}$; and ii) the $\mathrm{p} \dot{\mathrm{VO}}$, slow component amplitude increased by approximately $4 \%$ when expressed relative to the end-exercise $p \dot{V} O_{2}$. These results suggest an age-dependent effect on the physiological mechanisms regulating oxidative metabolism at the onset of heavy-intensity cycling exercise in males between the ages of 14-16 yrs.

\section{Phase II $p \dot{p V O}_{2}$ Response}

Data from the current study demonstrate a similar trend in the phase II $\tau$ to those previously reported from a longitudinal investigation of prepubertal children at the onset of heavy-intensity cycling exercise (13). Using the same apparatus, methods and analytical techniques over the same time period as the current study, Fawkner and Armstrong showed that in a group of 13 prepubertal boys (mean age $10.6 \pm$ $0.3 \mathrm{yr}$ ) the phase II $\tau$ significantly increased from $17 \pm 5 \mathrm{~s}$ to $22 \pm 5 \mathrm{~s}$ over a 2 -yr interval (13). In the current study the phase II $\tau$ significantly increased from 25 $\pm 5 \mathrm{~s}$ to $30 \pm 5 \mathrm{~s}$ over a 2 -yr interval, evidencing a progressively slower phase II response to heavy-intensity exercise with increasing age.

On the first test occasion with the younger boys we were able to assess pubic hair to estimate stage of maturation but because of the prevailing sociological climate in the UK at the time of other tests in the series we were unable to repeat the procedure on subsequent occasions. In an earlier longitudinal study of young people in the same age range as the present participants, we demonstrated an independent and positive maturational influence on peak $p \dot{V} O_{2}$ even with age, body size and fatness controlled for (3) but we and others have documented that, as in 
the current study, at the onset of heavy-intensity exercise there is no relationship between young people's peak $p \dot{V} O_{2}$ and either the primary $\tau$ or the slow component $(10,13)$. It is possible, however, that maturational changes have an independent influence on the $\tau$ and/or slow component over and above those due to chronological age, but this awaits further investigation. Adult data were not collected in the current study and inter study comparisons of $p \dot{V} \mathrm{O}_{2}$ kinetic studies must be made with extreme caution but it is of interest to note that the mean phase II $\tau$ obtained on the second test occasion in the current study is similar to values previously reported in adults (27) and in 14-17 yr olds (24) during exercise within the same exercise intensity domain.

It is not readily apparent why the phase II $\tau$ slows with age during the teen years but the primary response is considered to be principally dependent upon the mitochondrial potential to generate the required ATP for exercise, with a possible contribution from the efficiency to deliver oxygen to the site of utilization (2). The extent to which oxygen delivery limits the phase 2 response to heavy-intensity exercise is clouded by contradictory data $(19,20)$ and it seems most likely that oxygen delivery only becomes limiting when severely restricted by disease or old age (16). There is limited evidence of a tendency for oxygen delivery (muscle blood flow per unit tissue) to decrease from the ages of 12-16 yr (22) but whether oxygen delivery decreases with age and limits the kinetic response to heavy-intensity exercise during childhood remains to be determined.

There are several competing theories for the control of oxidative phosphorylation at the onset of exercise [see Poole et al. (28) for a recent review]. A popular contention, however, is that the rate of $p \dot{V} \mathrm{O}_{2}$ at exercise onset is regulated by the exchange of intramuscular phosphates between the splitting of ATP at the myofibrils and its subsequent synthesis at the mitochondria $(25,33)$. Upon a step increase in metabolic demand, ATP levels are temporally buffered by the breakdown of muscle $\mathrm{PCr}$ catalyzed by the creatine kinase reaction:

$$
\mathrm{PCr}+\mathrm{ADP}+\mathrm{H}^{+} \leftrightarrow \mathrm{ATP}+\mathrm{Cr}
$$

The subsequent release of creatine $(\mathrm{Cr})$ and its rephosphorylation by creatine kinase within the mitochondria provides the requisite ADP substrate that stimulates oxidative phosphorylation, and is termed the 'PCr-Cr shuttle' mechanism (9). Rossiter et al. (31) reported a dynamic symmetry between the rate of PCr breakdown and the rise of phase II $p \dot{V} O_{2}$ during the on-transient response in adults at the onset of high-intensity exercise. This suggests that the faster phase II $\mathrm{p} \dot{V} \mathrm{O}_{2}$ kinetics reported in children compared with adults might be explained by an age dependent change in putative phosphate linked controller(s) of mitochondrial oxidative phosphorylation. Whether there is an age-dependent effect on the exchange of intramuscular phosphates regulating mitochondrial oxidative phosphorylation during heavy-intensity exercise is presently unclear. However, a recent study from our laboratory, published in abstract form, found faster muscle PCr kinetics in $13 \mathrm{yr}$ old boys compared with men at the onset of heavy-intensity quadriceps exercise ( $\operatorname{PCr} \tau$ : 31 vs. $45 \mathrm{~s}$ respectively) (35), which is consistent with the $p \dot{V} O_{2}$ kinetics results presented in the current study. The mechanisms underlying the more rapid muscle PCr kinetics in children await resolution, although enhanced mitochondrial properties and/or lower stores of resting muscle PCr have been implicated (2). 


\section{p $\mathrm{VO}_{2}$ Slow Component}

As hypothesized, for exercise above the GET, we found a demonstrable $p \dot{V} O_{2}$ slow component in boys exercising within the heavy-intensity exercise domain. This supports the existence of a slow component of $\mathrm{p} \dot{\mathrm{V}} \mathrm{O}_{2}$ which was evidenced previously in prepubertal children $(12,13,15)$ and adolescent participants $(24)$ exercising in this domain. The relative contribution of the $p \dot{V} O$, slow component to the total gain in $p \dot{V} O_{2}$ increased significantly over the 2 -yr interval from $9 \pm 5 \%$ to 13 $\pm 4 \%$ on test occasions one and two respectively (see Figure 1). As stated earlier, cross-study comparisons should only be made cautiously but, it is pertinent to note that both the absolute and relative magnitude of the $p \dot{V} O_{2}$ slow component $(0.28$ $\pm 0.10 \mathrm{~L} \cdot \mathrm{min}^{-1}$ and $13 \pm 4 \%$ respectively) found on test occasion 2 were similar to those previously reported by Özeyner et al. (27) in adults (age $30.7 \pm 14.5 \mathrm{yr}$ ) during heavy-intensity cycling exercise $\left(0.22 \mathrm{~L} \cdot \mathrm{min}^{-1}\right.$ and $12 \%$ respectively). In the current study, the development of a slow component elevated the end exercise $p \dot{V} O_{2}$ gain to $12.4 \pm 0.7 \mathrm{~mL} \cdot \mathrm{min}^{-1} \cdot \mathrm{W}^{-1}$ on test occasion 2 which is also comparable to previously reported adult data (27).

The precise magnitude of the $p \dot{V} O_{2}$ slow component found on test occasion 2 in the current study is similar to that previously reported by Lai et al. (24) in similarly aged boys. However, in contrast to the double-exponential model used by Lai et al. (24), we opted to fit a single exponential model to data within a predetermined fitting window to estimate the phase II parameters, and computed the slow component as the difference between the phase II amplitude and end-exercise $p \dot{V} O_{2}$. It is of interest to note than when applying a double-exponential model to the current data on test occasion 2 , the mean $95 \%$ confidence intervals for the phase II $\tau$ increased from $\pm 4.8 \mathrm{~s}$ to $\pm 8.2 \mathrm{~s}$ respectively and resulted in 13 participants obtaining confidence intervals greater than $\pm 5 \mathrm{~s}$. This not only highlights the limitations of cross study comparisons but also reinforces concerns we have examined empirically and reported elsewhere with combining exponential terms within a single model when attempting to estimate accurately young people's responses to the onset of heavy-intensity exercise (14).

In combination with our previous investigation (13), the results from the current study indicate an age-related increase in the magnitude of the $p \dot{V} O_{2}$ slow component between 11 and 16 yrs of age. It is widely accepted that the additional $\mathrm{O}_{2}$ cost of exercise which manifests itself as the $p \dot{V} O_{2}$ slow component originates from processes occurring within the contracting muscle (18), and is temporally correlated with a greater muscle $\mathrm{PCr}$ cost of exercise (31). Previous studies have demonstrated that the emergence of the $p \dot{V} O_{2}$ slow component coincides both temporally and in magnitude with markers of muscle activity $(11,32)$, supporting the notion that augmented muscle recruitment patterns underlie the development of the $p \dot{V} \mathrm{O}_{2}$ slow component. A significant correlation between the percentage of type II muscle fibers inferred from biopsy data and amplitude of the $p \dot{V} O_{2}$ slow component lends further support to this hypothesis $(5,29)$. Likewise, the selective glycogen depletion of type I fibers results in slower phase II $p \dot{V} O_{2}$ kinetics and an elevated $p \dot{V} \mathrm{O}_{2}$ slow component when recruitment of type II fibers are presumably isolated during subsequent heavy-intensity exercise (23).

Given the above, it is possible that developmental changes in the expression of muscle fiber types at rest and/or their recruitment patterns during exercise may 
underlie the age-related increase in the $\mathrm{pV}_{2}$ slow component during heavy-intensity exercise. In a comprehensive review, Jansson (21) concluded that from the age of $10 \mathrm{yrs}$ the percentage of type 1 fibers decreases from $~ 58 \%$ to $48 \%$ circa 20 yrs of age in boys. Mizuno et al. (26) reported that during incremental forearm exercise, the increase in $\mathrm{P}_{\mathrm{i}} / \mathrm{PCr}, \mathrm{P}_{\mathrm{i}}$ and fall in $\mathrm{pH}$ were associated with a reduced expression of type I muscle fibers. In this sense, it is pertinent to note that children have also been reported to be characterized by a reduced increase in $\mathrm{P} / \mathrm{PCr}, \mathrm{Pi}$ and fall in $\mathrm{pH}$ during high intensity exercise compared with adults (37). A logical hypothesis therefore, is that during heavy-intensity exercise younger children achieve the required power output via a more 'homogeneous' recruitment of highly oxidative type I muscle fibers, which attenuates the accumulation of fatigue inducing metabolites $\left(\mathrm{P}_{\mathrm{i}}\right.$ and $\left.\mathrm{H}^{+}\right)$within the contracting muscle, and thus reduces the need to recruit less efficient type II motor units (2).

Data from animal models $(6,8)$ demonstrate that type II muscle fibers possess both microvascular and metabolic properties that limit the muscle cells' capacity to receive and use oxygen and rely more heavily on substrate-level phosphorylation. The additional recruitment of these muscle fibers during heavy-intensity exercise might be expected to increase the overall phosphate cost of force production and elevate the eventual $p \dot{V} \mathrm{O}_{2}$ gain. This trend is illustrated in Figure 2 for data from test occasion 2 compared with the first test occasion. Therefore, these data, coupled with previous evidence of an age-related decline in the expression of type I muscle fibers (21), might explain the increased $p \dot{V} O_{2}$ slow component and elevated total $p \dot{V} \mathrm{O}_{2}$ gain found over a 2-yr interval in the present investigation. However, this hypothesis requires further confirmation using models such as work-to-work exercise transitions (34) to manipulate muscle fiber recruitment patterns in participants at different stages of maturation.

\section{Conclusion}

In summary, this is the first investigation to examine longitudinal changes in $p \dot{V} O_{2}$ kinetics at the onset of heavy-intensity exercise in teenage boys. We found a significantly slower phase II $\tau$ and a greater $p \dot{V} O_{2}$ slow component over a 2 -yr interval and it is proposed that these results might be explained by an age dependent change in putative phosphate linked controller(s) of mitochondrial oxidative phosphorylation, and/or differences in muscle fiber recruitment patterns.

\section{Note}

The work was carried out in the Children's Health and Exercise Research Centre, School of Sport and Health Sciences, University of Exeter, Exeter, EX1 2LU, UK. Dr Fawkner is now with the School of Life Sciences, Heriot-Watt University, Riccarton, Edinburgh, EH14 4AS, UK.

\section{Acknowledgment}

We gratefully acknowledge the Community Fund, the Darlington Trust and the Healthy Heart Research Trust for their financial support of this study. 


\section{References}

1. Armon, Y., D.M. Cooper, R. Flores, S. Zanconato, and T.J. Barstow. Oxygen uptake dynamics during high-intensity exercise in children and adults. J. Appl. Physiol. 70:841-848, 1991.

2. Armstrong, N., and A.R. Barker. Oxygen uptake kinetics in children and adolescents: a review. Pediatr. Exerc. Sci. 21: 130-147, 2009.

3. Armstrong, N., and J.R. Welsman. Peak oxygen uptake in relation to growth and maturation. Eur. J. Appl. Physiol. 85: 546-551, 2001.

4. Barker, A.R., J.R. Welsman, J. Fulford, D. Welford, C.A. Williams, and N. Armstrong. Muscle phosphocreatine and pulmonary oxygen uptake kinetics in children at the onset and offset of moderate intensity exercise. Eur. J. Appl. Physiol. 102:727-738, 2008.

5. Barstow, T.J., A.M. Jones, P.H. Nguyen, and R. Casaburi. Influence of muscle fiber type and pedal frequency on oxygen uptake kinetics of heavy exercise. J. Appl. Physiol. 81:1642-1650, 1996.

6. Behnke, B.J., P. McDonough, D.J. Padilla, T.I. Musch, and D.C. Poole. Oxygen exchange profile in rat muscles of contrasting fibre types. J. Physiol. 549:597-605, 2003.

7. Bessman, S.P., and P.J. Geiger. Transport of energy in muscle: the phosphorylcreatine shuttle. Science. 211:448-452, 1981.

8. Bottinelli, R., and C. Reggiani. Human skeletal muscle fibres: molecular and functional diversity. Prog. Biophys. Mol. Biol. 73:195-262, 2000.

9. Carter, H., A.M. Jones, T.J. Barstow, M. Burnley, C.A. Williams, and J.H. Doust. Oxygen uptake kinetics in treadmill running and cycle ergometry: a comparison. $J$. Appl. Physiol. 89:899-907, 2000.

10. Cleuziou, C., A.M. Lecoq, R. Candau, D. Courteix, P. Guenon, and P. Obert. Kinetics of oxygen uptake at the onset of moderate and heavy exercise in trained and untrained prepubertal children. Sci. Sports. 17:291-296, 2002.

11. Endo, M.Y., M. Kobayakawa, R. Kinugasa, et al. Thigh muscle activation distribution and pulmonary $\mathrm{VO}_{2}$ kinetics during moderate, heavy, and very heavy intensity cycling exercise in humans. Am. J. Physiol. Regul. Integr. Comp. Physiol. 293:R812-R820, 2007.

12. Fawkner, S.G., and N. Armstrong. The slow component response of $\mathrm{VO}_{2}$ to heavy intensity exercise in children. In: Kinanthropometry VIII, T. Reilly and M. MarfellJones (Eds.). London: Routledge, 2003, pp. 105-113.

13. Fawkner, S.G., and N. Armstrong. Longitudinal changes in the kinetic response to heavy-intensity exercise in children. J. Appl. Physiol. 97:460-466, 2004.

14. Fawkner, S.G., and N. Armstrong. Modelling the $\mathrm{VO}_{2}$ kinetic response to heavy intensity exercise in children. Ergonomics. 47:1517-1527, 2004.

15. Fawkner, S.G., and N. Armstrong. Sex differences in the oxygen uptake kinetic response to heavy-intensity exercise in prepubertal children. Eur. J. Appl. Physiol. 93:210-216, 2004.

16. Fawkner, S.G., and N. Armstrong. Oxygen Uptake Kinetics. In: Paediatric Exercise Science and Medicine, N. Armstrong and W. Van Mechelen (Eds.). Oxford: Oxford University Press, 2008, pp. 297-307.

17. Fawkner, S.G., N. Armstrong, D.J. Childs, and J.R. Welsman. Reliability of the visually identified ventilatory threshold and V-slope in children. Pediatr. Exerc. Sci. 14:181-193, 2002.

18. Gaesser, G.A., and D.C. Poole. The slow component of oxygen uptake kinetics in humans. Exerc. Sport Sci. Rev. 24:35-71, 1996.

19. Grassi, B. Limitation of skeletal muscle $\mathrm{VO}_{2}$ kinetics by inertia of cellular respiration. In: Oxygen Uptake Kinetics in Sport, Exercise and Medicine, A.M. Jones and D.C. Poole (Eds.). London: Routeledge, 2005, pp. 212-229. 
20. Hughson, R.L. Regulation of $\dot{\mathrm{V}}_{2}$ kinetics by oxygen delivery. In: Oxygen Uptake Kinetics in Sport, Exercise and Medicine, A.M. Jones and D.C. Poole (Eds.). London: Routeledge, 2005, pp. 185-211.

21. Jansson, E. Age-related fiber type changes in human skeletal muscle. In: Biochemistry of Exercise IX, R. Maughan and S. Shirreffs (Eds.). Champaign, IL: Human Kinetics, 1996, pp. 297-307.

22. Koch, G. Aerobic power, lung dimensions, ventilatory capacity and muscle blood flow in 12-16 year old boys with high physical activity. In: Children and Exercise IX, K. Berg and B.O. Eriksson (Eds.). Baltimore MD: University Park Press, 1980, pp. 99-108.

23. Krustrup, P., K. Soderlund, M. Mohr, and J. Bangsbo. The slow component of oxygen uptake during intense, sub-maximal exercise in man is associated with additional fibre recruitment. Pflugers Arch. 447:855-866, 2004.

24. Lai, N., M.M. Nasca, M.A. Silva, F.T. Silva, B.J. Whipp, and M.E. Cabrera. Influence of exercise intensity on pulmonary oxygen uptake kinetics at the onset of exercise and recovery in male adolescents. Appl. Physiol. Nutr. Metab. 33:107-117, 2008.

25. Meyer, R.A. A linear model of muscle respiration explains monoexponential phosphocreatine changes. Am. J. Physiol. Cell Physiol. 254:C548-C553, 1988.

26. Mizuno, M., N.H. Secher, and B. Quistorff. ${ }^{31}$ P-NMR spectroscopy, rsEMG, and histochemical fiber types of human wrist flexor muscles. J. Appl. Physiol. 76:531-538, 1994.

27. Özyener, F., H.B. Rossiter, S.A. Ward, and B.J. Whipp. Influence of exercise intensity on the on- and off-transient kinetics of pulmonary oxygen uptake in humans. J. Physiol. 533:891-902, 2001.

28. Poole, D.C., T.J. Barstow, P. McDonough, and A.M. Jones. Control of oxygen uptake during exercise. Med. Sci. Sports Exerc. 40:462-474, 2008.

29. Pringle, J.S., J.H. Doust, H. Carter, K. Tolfrey, I.T. Campbell, and A.M. Jones. Oxygen uptake kinetics during moderate, heavy and severe intensity "submaximal" exercise in humans: the influence of muscle fibre type and capillarisation. Eur. J. Appl. Physiol. 89:289-300, 2003.

30. Rossiter, H.B., S.A. Ward, V.L. Doyle, F.A. Howe, J.R. Griffiths, and B.J. Whipp. Inferences from pulmonary $\mathrm{O}_{2}$ uptake with respect to intramuscular [phosphocreatine] kinetics during moderate exercise in humans. J. Physiol. 518(Pt 3):921-932, 1999.

31. Rossiter, H.B., S.A. Ward, F.A. Howe, J.M. Kowalchuk, J.R. Griffiths, and B.J. Whipp. Dynamics of intramuscular ${ }^{31} \mathrm{P}-\mathrm{MRS} \mathrm{P}_{\mathrm{i}}$ peak splitting and the slow components of $\mathrm{PCr}$ and $\mathrm{O}_{2}$ uptake during exercise. J. Appl. Physiol. 93:2059-2069, 2002.

32. Saunders, M.J., E.M. Evans, S.A. Arngrimsson, J.D. Allison, G.L. Warren, and K.J. Cureton. Muscle activation and the slow component rise in oxygen uptake during cycling. Med. Sci. Sports Exerc. 32:2040-2045, 2000.

33. Walsh, B., M. Tonkonogi, K. Soderlund, E. Hultman, V. Saks, and K. Sahlin. The role of phosphorylcreatine and creatine in the regulation of mitochondrial respiration in human skeletal muscle. J. Physiol. 537:971-978, 2001.

34. Wilkerson, D.P., and A.M. Jones. Effects of baseline metabolic rate on pulmonary $\mathrm{O}_{2}$ uptake on-kinetics during heavy-intensity exercise in humans. Respir. Physiol. Neurobiol. 156:203-211, 2007.

35. Willcocks, R.J., A.R. Barker, J. Fulford, et al. Kinetics of phosphocreatine and deoxyhemoglobin in children and adults during high-intensity exercise (Abstract). Med. Sci. Sports Exerc. 40:S20, 2008.

36. Williams, C.A., H. Carter, A.M. Jones, and J.H. Doust. Oxygen uptake kinetics during treadmill running in boys and men. J. Appl. Physiol. 90:1700-1706, 2001.

37. Zanconato, S., S. Buchthal, T.J. Barstow, and D.M. Cooper. ${ }^{31} \mathrm{P}-\mathrm{magnetic}$ resonance spectroscopy of leg muscle metabolism during exercise in children and adults. J. Appl. Physiol. 74:2214-2218, 1993. 